\title{
A Remarkable Property of Definite Integrals
}

\author{
By M. L. Glasser
}

Abstract. We show that the integral of $F(u)$ is the same as the integral of $F(x)$, both with respect to $x$, for a variety of functions $u$.

In this note $F$ will denote any integrable function, and all integral signs denote the Cauchy principal value. The formula

$$
I=\int_{-\infty}^{\infty} F(u) d x=\int_{-\infty}^{\infty} F(x) d x
$$

where

$$
u=x-1 / x,
$$

goes back at least as far as Cauchy and can be proved as follows: From a graph of the relation (2) one sees that the solution for $x$ has two branches

$$
x_{+}=\frac{1}{2}\left\{u \pm \sqrt{u^{2}+4}\right\}, \quad-\infty<u<\infty .
$$

Thus

$$
I=\int_{-\infty}^{0-} F(u) d x_{-}+\int_{0^{+}}^{\infty} F(u) d x_{+}=\int_{-\infty}^{\infty} F(u)\left(x_{-}^{\prime}+x_{+}^{\prime}\right) d u .
$$

But

$$
x_{-}^{\prime}+x_{+}^{\prime}=\frac{1}{2}\left\{1-\frac{u}{\sqrt{u^{2}+4}}+1+\frac{u}{\sqrt{u^{2}+4}}\right\}=1 .
$$

Thus, for example,

$$
\begin{aligned}
\int_{0}^{\infty} \exp \left(-x^{2}-1 / x^{2}\right) d x & =\frac{1}{2 e^{2}} \int_{-\infty}^{\infty} \exp \left(-(x-1 / x)^{2}\right) d x \\
& =\frac{1}{2 e^{2}} \int_{-\infty}^{\infty} e^{-x^{2}} d x=\frac{\sqrt{\pi}}{2 e^{2}} .
\end{aligned}
$$

Equations (1), (2) clearly represent a useful property of the definite integral that deserves to be widely known.

What is more remarkable is that (1) remains true when (2) is replaced by

$$
u=x-\sum_{j=1}^{n-1} \frac{a_{j}}{x-C_{j}}
$$

Received June 21, 1982.

1980 Mathematics Subject Classification. Primary 26A06, 26 A42. 
where $\left\{a_{j}\right\}$ is any sequence of positive constants and the $C_{j}$ are any real constants whatsoever.

The proof for (3), as can be seen by drawing a graph, is a simple extension of that for (2). Without loss of generality we can assume that $C_{1}<C_{2}<C_{3}<\ldots$ Equation (3) reduces to an $n$th degree equation for $x$ of the form

$$
x^{n}-\left(u-\sum_{j=1}^{n-1} C_{j}\right) x^{n-1}+\cdots=0 .
$$

Its solution has $n$ branches, for each of which $-\infty<u<\infty$, such that

$$
x_{1}+\cdots+x_{n}=u-\sum_{j=1}^{n-1} C_{j} \text {. }
$$

Hence

$$
x_{1}^{\prime}+\cdots+x_{n}^{\prime}=1
$$

Thus

$$
\begin{aligned}
I & =\left(\int_{-\infty}^{C_{1}^{-}} d x_{1}+\int_{C_{1}^{+}}^{C_{2}^{-}} d x_{2}+\cdots+\int_{C_{n-1}^{+}}^{\infty} d x_{n}\right) F(u) \\
& =\int_{-\infty}^{\infty} F(u)\left(x_{1}^{\prime}+\cdots+x_{n}^{\prime}\right) d u=\int_{-\infty}^{\infty} F(u) d u .
\end{aligned}
$$

The same idea applies to any function of $x$ whose inverse breaks up into homologous branches. Futhermore, there is no difficulty in letting $n \rightarrow \infty$, as long as the series in (3) converges. Thus from the partial fraction decomposition for the cotangent we find that (1) is valid for

$$
u=x-\sum_{j} a_{j} \cot \left[\left(x-C_{j}\right)^{-1}\right] .
$$

When not all the branches are real, as for $u=x^{3}-1 / x$, the problem is more difficult and is under investigation. The case

$$
w=\sum_{j=1}^{n} \frac{a_{j}}{x+C_{j}}
$$

is interesting, for $x$ satisfies the equation

$$
w x^{n}-\left(w \sum C_{j}+\sum a_{j}\right) x^{n-1}+\cdots=0,
$$

and there should be $n$ branches. However, the graph apparently shows $n+1$ branches. The resolution is that for the first solution $x_{1}, 0>w>-\infty$, and for the last, $x_{n}, \infty>w>0$. Thus these portions of the graph combine to form a single branch $x_{1}$ for which $-\infty<w<\infty$. Now, noting that

$$
x_{1}+\cdots+x_{n}=\sum C_{j}+\frac{1}{w} \sum a_{j}
$$

so

$$
x_{1}^{\prime}+\cdots+x_{n}^{\prime}=-\sum a_{j} / w^{2}
$$

we have as before, after a simple change of variable,

$$
\int_{-\infty}^{\infty} F(w) d x=\sum a_{j} \int_{-\infty}^{\infty} F(1 / x) d x
$$


For example $F(x)=x^{2} /\left(x^{2}+1\right), w=(x+a)^{-1}+(x+b)^{-1}$ gives

$$
\int_{-\infty}^{\infty} \frac{(2 x+a+b)^{2} d x}{(x+a)^{2}(x+b)^{2}+(2 x+a+b)^{2}}=2 \pi
$$

independently of $a$ and $b$.

The independence of (1) and (6) of the various constants appearing in (3) and (5) is interesting and leads to the following "paradox".

It is an elementary exercise to show that for $a \neq 0$ :

(i) $\int_{-\infty}^{\infty} F\left(x-a^{2} / x\right) d x / x=0$,

(ii) $\int_{-\infty}^{\infty} F\left(x-a^{2} / x\right) d x / x^{2}=\left(1 / a^{2}\right) \int_{-\infty}^{\infty} F(x) d x$. Now suppose that $G^{\prime}(x)=$ $F(x)$. Then from (i)

$$
\phi(a)=\int_{-\infty}^{\infty} G\left(x-a^{2} / x\right) \frac{d x}{x}=0
$$

Thus $\phi^{\prime}(a)=0$, i.e.

$$
0=-2 a \int_{-\infty}^{\infty} G^{\prime}\left(x-a^{2} / x\right) \frac{d x}{x^{2}}=-2 a \int_{-\infty}^{\infty} F\left(x-a^{2} / x\right) \frac{d x}{x^{2}},
$$

and by (ii) this becomes

$$
\int_{-\infty}^{\infty} F(x) d x=0
$$

Thus, the integral of any function with an antiderivative appears to vanish. The resolution of this disturbing result is that $G^{\prime}\left(x-a^{2} / x\right)$ is discontinuous at $x=0$ unless (8) is true, so that differentiation under the integral sign in (7) is allowed only in this case.

Finally it should be remarked that the function $F$ may be nonzero over a limited range so the limits of integration need not actually be infinite. Thus (6) with

$$
w=\frac{1}{2}\left\{\frac{1}{x-1}+\frac{1}{x+1}\right\}, \quad F(x)= \begin{cases}e^{-1 / x}, & x>0, \\ 0, & x \leqslant 0\end{cases}
$$

leads to the identity

$$
\int_{1}^{\infty} \exp \left[-\frac{1}{2}(x-1 / x)\right] d x=2-\int_{0}^{1} \exp \left[\frac{1}{2}(x-1 / x)\right] d x
$$

as can easily be proven directly.

The transformations discussed here and perhaps similar ones which remain to be worked out would seem to be invaluable when faced with the integration of complicated functions of rational expressions.

Acknowledgements. This work was supported by the National Science Foundation.

Department of Mathematics and Computer Science

Clarkson College of Technology

Potsdam, New York 13676 